\title{
Article \\ On the Specificity and Permanence of Electroencephalography Functional Connectivity
}

\author{
Yibo Zhang, Ming Li *, Hui Shen and Dewen Hu
}

check for updates

Citation: Zhang, Y.; Li, M.; Shen, H.; $\mathrm{Hu}, \mathrm{D}$. On the Specificity and

Permanence of

Electroencephalography Functional

Connectivity. Brain Sci. 2021, 11, 1266.

https://doi.org/10.3390/

brainsci11101266

Academic Editor: Stephen D. Meriney

Received: 16 August 2021

Accepted: 17 September 2021

Published: 24 September 2021

Publisher's Note: MDPI stays neutral with regard to jurisdictional claims in published maps and institutional affiliations.

Copyright: (c) 2021 by the authors. Licensee MDPI, Basel, Switzerland. This article is an open access article distributed under the terms and conditions of the Creative Commons Attribution (CC BY) license (https:/ / creativecommons.org/licenses/by/ $4.0 /)$.
College of Intelligence Science and Technology, National University of Defense Technology, Changsha 410073, China; zhangyibo@nudt.edu.cn (Y.Z.); shenhui@nudt.edu.cn (H.S.); dwhu@nudt.edu.cn (D.H.)

* Correspondence: liming78@nudt.edu.cn

\begin{abstract}
Functional connectivity, representing a statistical coupling relationship between different brain regions or electrodes, is an influential concept in clinical medicine and cognitive neuroscience. Electroencephalography-derived functional connectivity (EEG-FC) provides relevant characteristic information about individual differences in cognitive tasks and personality traits. However, it remains unclear whether these individual-dependent EEG-FCs remain relatively permanent across long-term sessions. This manuscript utilizes machine learning algorithms to explore the individual specificity and permanence of resting-state EEG connectivity patterns. We performed six recordings at different intervals during a six-month period to examine the variation and permanence of resting-state EEG-FC over a long period. The results indicated that the EEG-FC networks are quite subject-specific with a high-precision identification accuracy of greater than $90 \%$. Meanwhile, the individual specificity remained stable and only varied slightly after six months. Furthermore, the specificity is mainly derived from the internal connectivity of the frontal lobe. Our work demonstrates the existence of specific and permanent EEG-FC patterns in the brain, providing potential information for biometric applications.
\end{abstract}

Keywords: functional connectivity; permanence; individual specificity; EEG

\section{Introduction}

Brain functional connectivity plays an essential role in neuroscience. It reflects the complex functional dependence and coupling of neural activity between brain regions [1]. Measures of connectivity can be recognized by a variety of physiological recording techniques, including magnetic resonance imaging (MRI), near-infrared spectroscopy, and electroencephalography (EEG). As a new method of individual differences and pathological research, functional network analysis has attracted increasing attention from the scientific community $[2,3]$. However, the individual specificity and permanence of human brain networks have not been thoroughly studied.

Individuals may have unique and characteristic connectivity patterns. Some previous MRI studies have demonstrated that functional connectivity networks exhibit high variability among individuals, serving as "fingerprints" of individuals [4]. fMRI provides good anatomical resolution and endogenous explanations for individual differences in functional brain networks, but its temporal resolution is limited [5]. Unlike fMRI, EEG is a practical and convenient approach to explore the temporal changes in functional brain connectivity, non-invasively recording neuronal activity at the millisecond level [6].

The increasing application of EEG network research has attracted attention regarding whether EEG functional connectivity (EEG-FC) can be sustained over time and across cognitive states. Permanence represents the intraindividual stability of brain activities over a period of time [7]. Previous studies, such as those on EEG biometrics, only dealt with single-session data sets. This study design leads to a concern about whether the accuracy of the recognition represented the uniqueness of the recognition according to the EEG characteristics of the subjects or the uniqueness of each acquisition session [8]. At 
present, some studies have begun to focus on the permanence of biometrics $[9,10]$. A study found that the permanence of the core-specific network structure of EEG-FC in five healthy subjects remained stable for several days [11]. Some studies have demonstrated that restingstate EEG-FC represents a powerful method for high-precision biometric identification purposes $[6,12]$.

However, these studies on EEG-FC patterns recorded only two or three sessions over a few weeks, which resulted in a lack of variation over a long period in the longitudinal study. Therefore, it is necessary to establish a sufficient number of recordings to firmly demonstrate whether specific functional connectivity can remain relatively permanent across time. In this manuscript, we explored the individual specificity and temporal permanence of resting-state EEG-FC from 15 healthy participants with multiple experimental sessions over six months.

Based on the previous fMRI and EEG studies, we had two hypotheses: (1) There may be individual differences in EEG-FC patterns, which could be verified by identification. (2) The individual-specific EEG-FC may be relatively permanent across disjoint sessions. Biometric identification was used as a research strategy in this manuscript to verify these hypotheses. Our exploratory work may be helpful to reveal the individual differences in neural electrical signal activity.

\section{Materials and Methods}

\subsection{Participants}

A total of fifteen healthy subjects ( $24 \pm 2$ years old) participated in this study. No history of neurological or psychiatric disorders, migraine, diabetes, or tinnitus was reported. All participants were right-handed and had normal vision (or corrected vision). All participants submitted written informed consent after receiving a detailed explanation of the experimental procedure. The studies involving human participants were reviewed and approved by the Medical Ethics Committee of 921 Hospital.

\subsection{Experiment Protocol}

The experimental environment was quiet. As shown in Figure 1, the subjects were given experimental precautions and sat in a comfortable chair $1 \mathrm{~m}$ away from the monitor. To reduce eye movement, the participants were required to look at a single black ' + ' in the center of a display screen for at least $2 \mathrm{~min}$ and close eyes for another $2 \mathrm{~min}$. During the recording of the 4 min resting-state EEG, the participants needed to keep still, avoid blinking, and to not think about other things. Recordings were repeated multiple times for each subject over a period of six months, including Day 1 (Session A), Day 2 (Session B), Day 7 (Session C), Day 30 (Session D), Day 90 (Session E), and Day 180 (Session F).
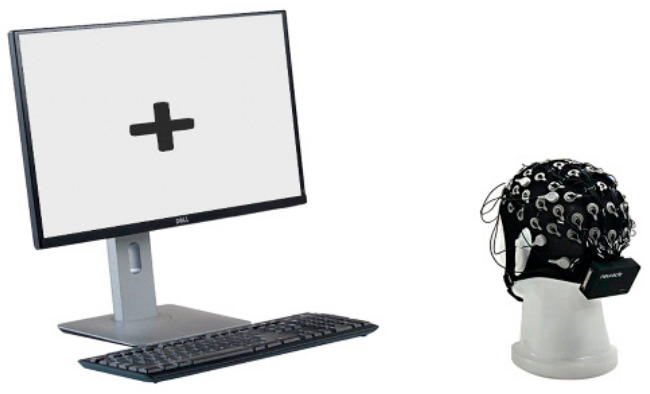

Figure 1. EEG Acquisition.

\subsection{Data Acquisition and Pre-Processing}

In the process of creating the dataset, we used Nerusen 32-channel wireless EEG acquisition equipment from the Neuracle company(Changzhou, China). The position of the electrodes was shown in Figure 2. According to the 10/20 international standard system, the electrodes were located at frontal, central, temporal, parietal, and occipital 
scalp sites [13] (Fp1, Fp2, F7, F3, Fz, F4, F8, FC5, FC1, FC2, FC6, A1, T7, T8, A2, C3, Cz, $\mathrm{C} 4, \mathrm{CP} 5, \mathrm{CP} 1, \mathrm{CP} 2, \mathrm{CP} 6, \mathrm{P} 7, \mathrm{P} 3, \mathrm{Pz}, \mathrm{P} 4, \mathrm{P} 8, \mathrm{PO} 3, \mathrm{PO} 4, \mathrm{O} 1, \mathrm{Oz}$, and O2). The reference electrodes were REF and GND. The sampling rate was set to $1000 \mathrm{~Hz}$, and the electrode impedance was adjusted below $10 \mathrm{k} \Omega$.

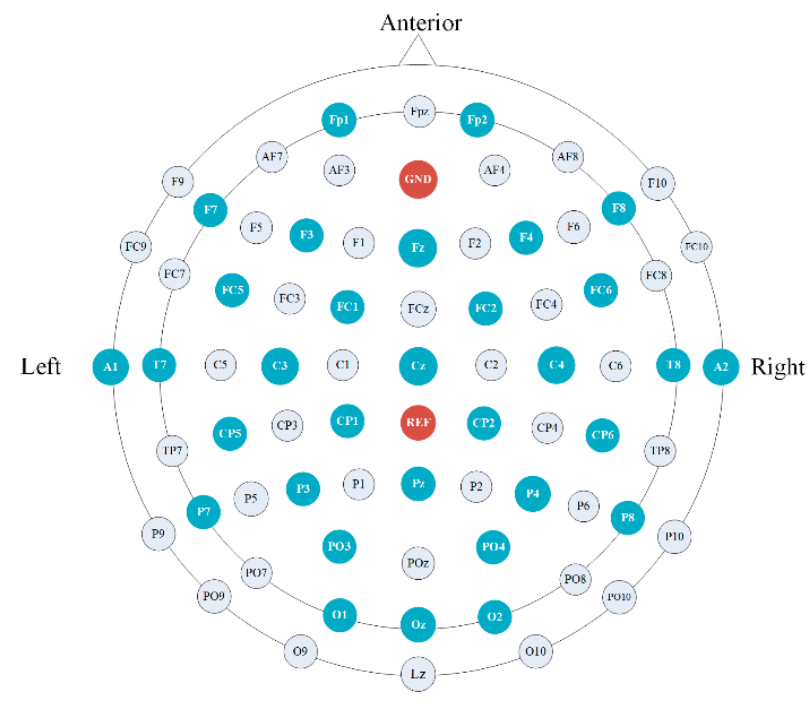

Figure 2. Electrode Placement.

EEG data were exported to EEGLAB [14] for pre-processing. In the first step, EEG data were filtered with a bandpass filter of $1-40 \mathrm{~Hz}$, including a notch filter of $50 \mathrm{~Hz}$. Then, eye movements, eyeblinks, muscle activity, and other obvious artifacts were corrected by applying an independent-component analysis (ICA) implemented in the EEGLab toolbox. Based on the artifact-free dataset (Reject ICs), we applied a spatial Laplacian filter using the current source density (CSD) toolbox to reduce the effects of volume conduction [15].

\subsection{Connectivity Measures and Machine Learning}

Previous studies have suggested that $2 \mathrm{~s}$ is sufficient for functional connectivity of resting-state EEG data [16]. Therefore, the preprocessed EEG data were segmented every two seconds. We performed the same process described above for each session dataset. Each session dataset included 15 subjects, 4 min of resting-state EEG signals. In total, this resulted in $120 \times 15$ segments for connectivity calculations. In order to maximize the retention of individual brain signal characteristics, we chose to retain the neural activity of all frequency bands for brain network analysis. The frequency band of EEG-FC was selected as 1-40Hz. We used the Hermes Toolbox [17] to calculate the Granger causality (GC) index and the mutual information (MI) index.

Granger causality, derived from the definition of causality in statistics, was introduced into neuroscience to describe brain functional connectivity. For two simultaneously measured time series signals $x(t)$ and $y(t), x(t)$ causes $y(t)$ if the former contains information that helps predict the future of the latter. Two time series $x(t)$ and $y(t)$ are modeled by BVAR (the bivariate autoregressive model), which including the past samples from the time series itself and the other time series. The calculation formula of the Granger causality index is as follows:

$$
\begin{aligned}
& x(n)=\sum_{k=1}^{P} a_{x \mid x, k} x(n-k)+\sum_{k=1}^{P} a_{x \mid y, k} y(n-k)+u_{x y}(n) \\
& y(n)=\sum_{k=1}^{P} a_{y \mid x, k} x(n-k)+\sum_{k=1}^{P} a_{y \mid y, k} y(n-k)+u_{y x}(n)
\end{aligned}
$$




$$
\begin{gathered}
V_{x \mid \bar{x}, \bar{y}}=\operatorname{var}\left(u_{x y}\right) \\
V_{y \mid \bar{x}, \bar{y}}=\operatorname{var}\left(u_{y x}\right) \\
G C_{y \rightarrow x}=\ln \left(\frac{V_{x \mid \bar{x}}}{V_{x \mid \bar{x}, \bar{y}}}\right)
\end{gathered}
$$

In Equations (1) and (2), $a_{i j}$ are the model parameters, $p$ is the order of the BVAR model and $u_{i}$ are the residuals associated to the model, $\operatorname{var}($.$) is the variance over time and$ $x \mid \bar{x}, \bar{y}$ is the prediction of $x(t)$ by the past samples of values of $x(t)$ and $y(t)$. Granger causality (GC) from $y(t)$ to $x(t)$ greater than or equal to zero. In our calculation, the model order $p$ was 15. The GC net relationship enabled the detection of directed and reciprocal influences common in brain coupling [18]. The GC net calculated by our recording data could describe the individual effective connectivity between distinct electrodes in the resting state.

Based on concepts from information theory, mutual information (MI) measures the interdependence between two variables, which quantifies the amount of information obtained from about one random variable by other random variables [19].

$$
M I_{x y}=\sum_{i} p(x, y) \log \frac{p(x, y)}{p(x) p(y)}
$$

In Equation (4), $p(x, y)$ is the joint probability distribution function of $x(t)$ and $y(t)$, and $p(x)$ and $p(y)$ are the marginal probability distribution functions of $x(t)$ and $y(t)$, respectively. The equation represents the cross-mutual information between $x(t)$ and $y(t)$. The MI of two random variables is a measure of the mutual dependence between the two variables. If the value of $\mathrm{MI}$ is zero, the electrical signals of the two channels were independent.

As shown in Figure 3, after constructing the brain network matrix of GC and MI, we used the identification procedures as a strategy evaluating individual specificity and permanence. We calculated that the number of net features between all electrode pairs of the GC index and MI index at each segment was $1024(32 \times 32)$ and $496(32 \times 31 / 2)$, respectively. As a reliable method, machine learning has been frequently used in previous MRI and EEG studies [20,21]. We used a support vector machine with a radial basis kernel function (SVM-RBF) algorithm to identify 15 participants. The one-against-one (OAO) multiclassification method was used in this manuscript. OAO designs a classifier between any two types of samples. When making classification decisions for an unknown sample, the voting method is adopted, and the category with the most votes in the category of the unknown sample.

We utilized the multi-classification method of machine learning for individual identification. By classifying the subject in a single session data set, we can explore whether the functional connectivity based on EEG could realize high variability among individuals. High-precision individual identification could indicate the existence of individual-specific brain network. In addition, the reproducibility and permanence of specific brain networks could be reflected by the classification accuracy across disjoint sessions. 


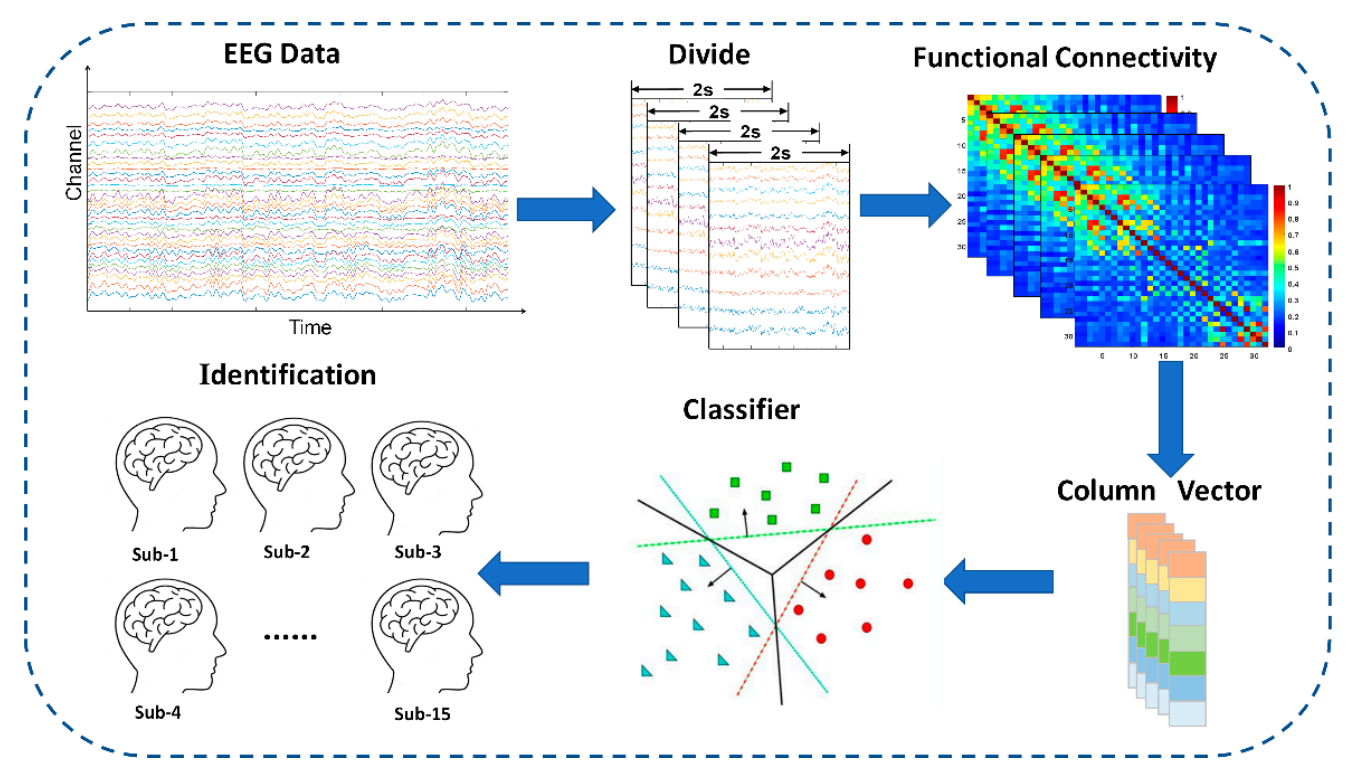

Figure 3. Illustration of identification flow.

\section{Results}

3.1. The Functional Connectivity Network between Individuals Is Specific within Different Sessions

The accuracy of identification in a single session represents the strength of individual specificity. High identification accuracy corresponds to strong individual functional connectivity differences. To explore the specificity of functional connectivity index, we performed identification classification on each session dataset. A total of 9/10 of each session dataset was taken as the training set, and 1/10 served as the testing set. Through ten-fold cross-validation, the 15-classification accuracy of each single session was obtained. Figure 4 shows multiple experimental sessions (A-F) conducted on the same group of subjects over a period of 6 months. The time points are Day 1, Day 2, Day 7, Day 30, Day 90, and Day 180. Figure 5a shows the individual specificity of functional connectivity from each single session experiment. The data of the same session experiment are divided into the training set and test set, and the classification accuracy is obtained by ten-fold cross-validation. The 15-classification accuracy of the GC network on a single session was 0.89 to 0.96 , and the MI network was 0.82 to 0.92 , respectively. Here, we show that individuals have unique EEG-FC profiles similar to fingerprints.

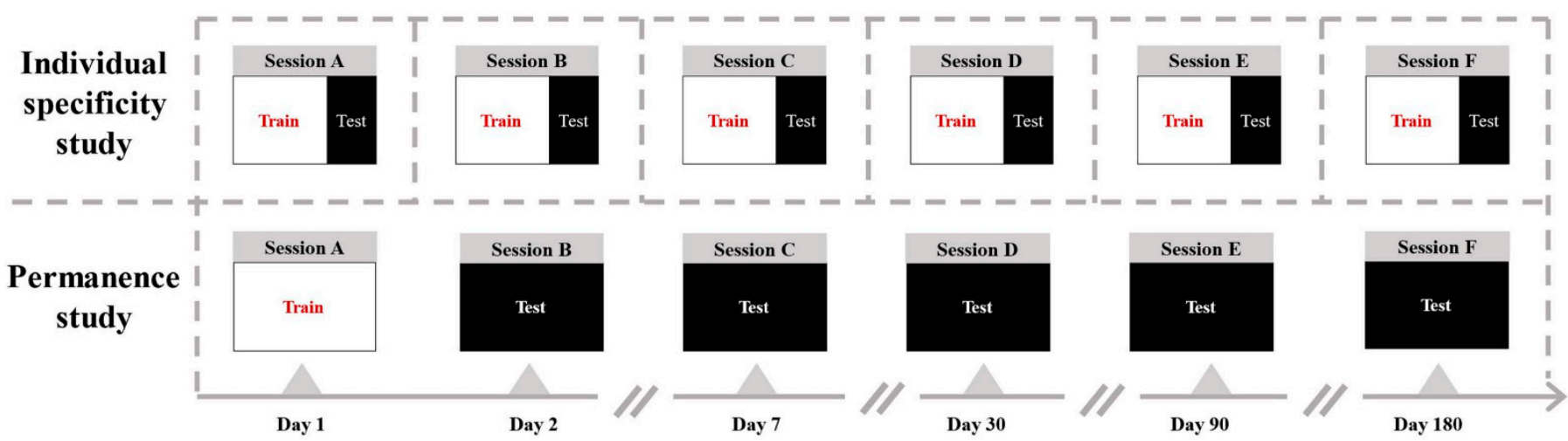

Figure 4. Illustration of individual specificity study in each single session and permanence study across different sessions. Sessions A to $\mathrm{F}$ represent EEG data acquired at 6 different time points over 6 months. The time points were Day 1, Day 2, Day 7, Day 30, Day 90, and Day 180. 
(a)

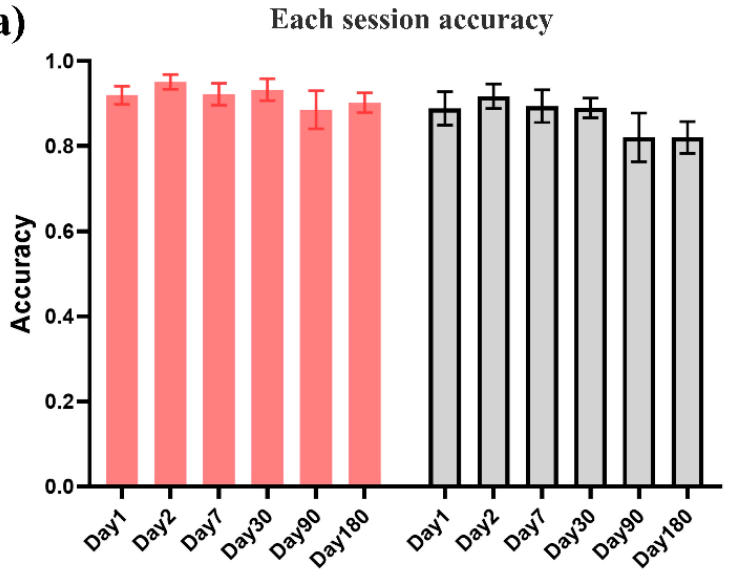

(b) Across sessions accuracy

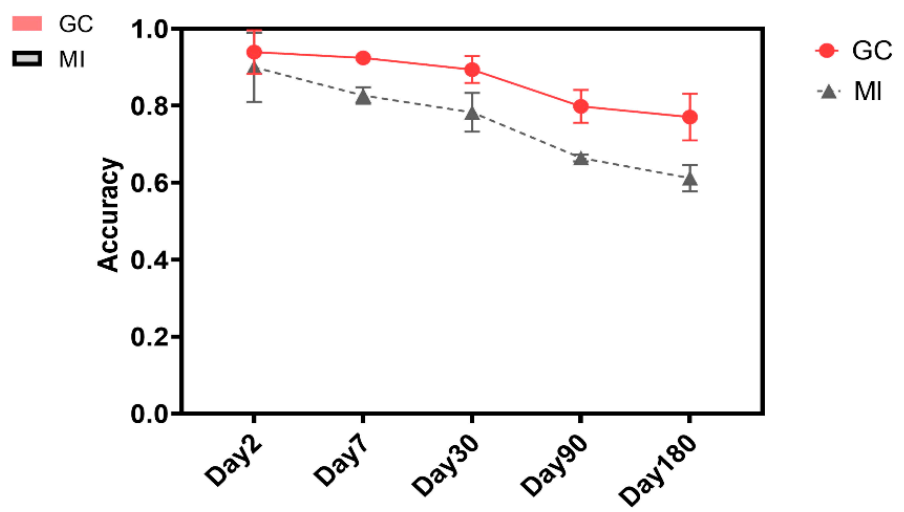

Figure 5. The classification result of intra-sessions (a) and inter-sessions (b). The identification accuracy of intra-sessions represented the specificity of the individual network, and the accuracy of intersessions represented the permanence of the brain functional network. (a) The intra-session identification accuracy. (b) The inter-session identification accuracy.

\subsection{Individuals Have Relatively Permanent Functional Connectivity Patterns across Long-Term Sessions}

The accuracy of identification across sessions represents the permanence of individual specificity. High accuracy corresponds to the similarity of individual functional connectivity between subsequent follow-up sessions and first sessions. To explore the permanence of the functional connectivity metric, we tested it across sessions. Session A collected on Day 1 was used as the training set. Session B collected on Day 2 was used as the testing set. The Session C-F data at other time points were used as the test set, in turn. The classification accuracy of cross-time sessions was obtained. Figure $5 \mathrm{~b}$ shows the permanence of functional connectivity. The data of the first-day session is used as the training set, and the data of other session experiments are used as the test set. The GC network classification accuracy decreased from 0.939 on the second day to 0.770 on the 180 th day. It shows that the individual specificity remained relatively stable and vary slightly in 6 months. The classification accuracy of mutual information decreased from 0.903 on the second day to 0.627 on the 180th day, which showing a monotonous decrease.

\subsection{The Difference in Individual Brain Networks Mainly Comes from the Internal Connections of Frontal Lobe}

According to the 10/20 international standard system, the electrodes were located at frontal, central, temporal, parietal, and occipital scalp sites [13]. EEG signals collected by different regions of the brain surface reflect completely different information. To explore the contribution of different brain regions to GC net specificity, we used machine learning to identify the features that make important contributions to classification. We extracted the classification scores of each feature vector and applied canonical correlation analysis (CCA) to find the related functional connectivity. The functional connectivity of each pair of electrodes was assigned a weight, indicating its contribution to the identification process. The major connection is defined as the functional connectivity with the top $10 \%$ of feature weights in the identification by SVM. The weight represents each pair's contribution to the identification process. Larger weights indicate more specific connections.

We used BrainNet [22] to draw the top view, right view, frontal view, and left view of the specific connectivity distributed on the brain surface. For clear representation, the colored lines indicate connections within each brain region, and grey lines represent inter-region connections. As shown in Figure 6, The larger spheres in the red network indicate a larger number of major connections in the frontal lobe region. Different colored spheres represent electrodes in different brain regions. Compared to other colors of the connection, these red lines indicate strong connections between electrodes in the frontal region of the brain. The difference in individual brain networks is mainly derived from 
the internal connections of the frontal lobe. The results indicated that individuals have relatively permanent specific connectivity patterns mainly due to the internal connections of the frontal lobe.

(a)

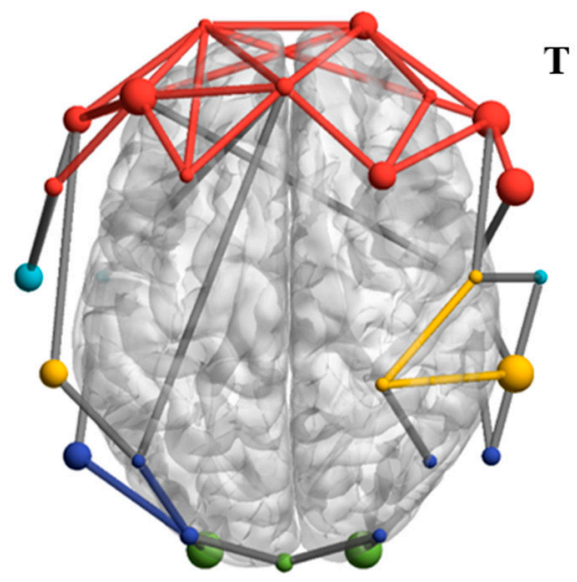

(c)

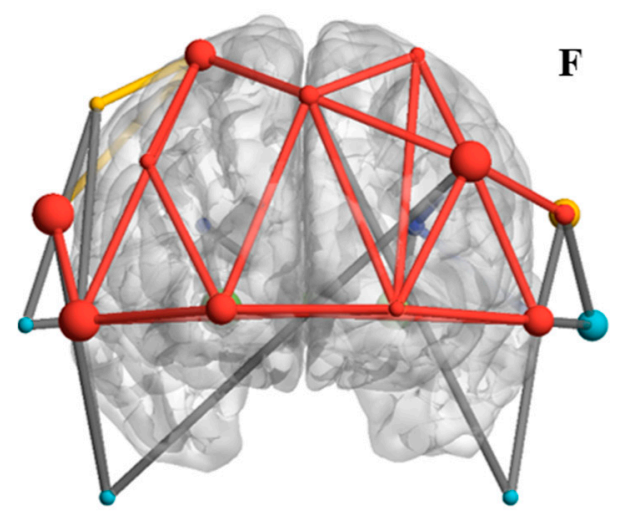

(b)

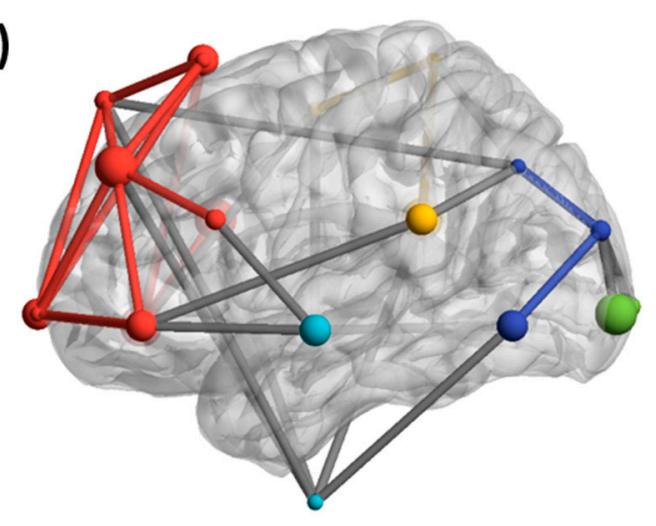

$\mathbf{L}$ (d)

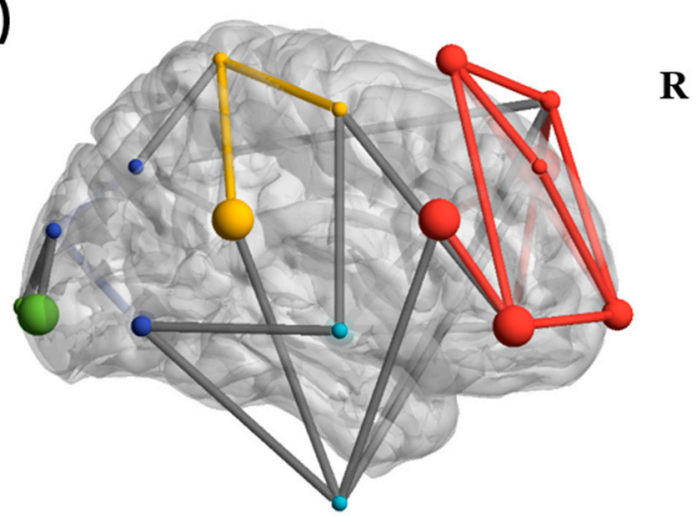

\section{Frontal $\bigcirc$ Temporal $\odot$ Parietal $\bigcirc$ Central $\bigcirc$ Occipital}

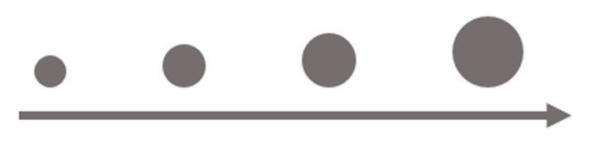

\section{Connectivity Number}

Figure 6. The major connections of individual EEG-FC networks. The size of the spheres represents the number of major connections to each electrode. Different colored spheres represent electrodes in different brain regions. The coloured lines indicate connections within each brain region, and grey lines represent inter-region connections. (a) Top view, (b) frontal view, (c) left view, and (d) right view.

\section{Discussion}

The neural activation and connectivity of non-invasive high-resolution spatiotemporal patterns have greatly improved our understanding of the individual mechanisms involved in perception, attention, and learning. Measures of functional connectivity of EEG signals are increasingly being used to study brain function in spontaneous neural activity. In our work, the brain network based on GC has the potential to reveal the long-term specific patterns of individuals. Our work shows that the measurement of EEG-FC at the sensor level can be used for biometric purposes with high recognition accuracy. In addition, the permanence of EEG-FC as a biological identifier was further demonstrated. Functional and effective connectivity measures convey important information about the neural network. 
Although the EEG network is generally believed to be fragile, it was demonstrated to be suitable for convenient and efficient biometrics.

Resting-state is a state in which the brain keeps quiet and awake without performing specific cognitive tasks. It may be the most basic but essential state of the brain [23]. Our results described the specificity and permanence of resting-state EEG connectivity measures, which could be considered in exploring inter-individual differences in brain relationships. However, it is important to note that this study only focused on the restingstate EEG, and caution is necessary when extending our results to event-related potentials or EEG of natural stimuli. It still needs to further explore whether specific EEG functional networks could maintain permanence under various cognitive tasks such as watching videos, playing games, and listening to music.

EEG signals collected from different areas of the brain surface reflect completely different neural activity information within the brain. Different brain regions could reflect different functional states [24]. Previous fMRI studies using data from the Human Connectome Project have demonstrated that the frontoparietal network emerged as most distinctive in individual characteristic connectivity patterns [4]. Although EEG does not have high-intensity spatial resolution, we could describe the correspondence of brain scalp functional areas through the electrodes on the surface scalp. The functional network consists of neural electrophysiological activity that can span multiple scalp regions of the brain [25]. We explored the major connections in the network by the contribution of each pair of electrodes to the recognition process. The frontal lobes represent higher cognitive functions and are responsible for learning, language, decision-making, and emotion [24,26]. Neural activity in the frontal lobe represents the region where conscious thoughts and decisions occur [27]. This feature may have resulted in a stable GC network in the flow of neuro-electrical signals to the frontal lobe network. In the process of investigating the EEG-FC, our conclusion verifies to some extent that the frontal network corresponding to EEG is also the most distinctive region of individual specificity in the resting state. This may help to represent individual brain networks with more significant and fewer EEG networks. In addition, electrical activity in the resting state may produce significant functional connectivity networks in various functional areas. EEG-FC patterns within frontal functional areas may be more assertive in the resting state. In the future study, it still needs to explore whether the permanence of individual EEG-FC patterns is independent of the various cognitive tasks across time.

\section{Conclusions}

In this study, we have explored the individual specificity and temporal permanence of EEG-FC with multiple experimental sessions over a relatively long time. Through our research, we report the following conclusions. The EEG-FC network based on Granger causality could exist for a long time as an individual unique connectivity pattern. In the characteristic connectivity pattern, the internal connections of the frontal lobe network may play a significant role. These results are conducive to revealing the individual differences in neural electrical signal activity and promote the application of the resting-state brain network in biometric identification.

Author Contributions: Conceptualization, M.L., H.S.; methodology, Y.Z., H.S.; software, Y.Z.; validation, M.L., D.H.; formal analysis, Y.Z., H.S.; investigation, Y.Z., M.L.; resources, M.L., D.H.; data curation, Y.Z., M.L.; writing-original draft preparation, Y.Z.; writing-review and editing, Y.Z., M.L., H.S.; visualization, Y.Z.; supervision, M.L., H.S. and D.H.; project administration, D.H.; funding acquisition, M.L., H.S. and D.H. All authors have read and agreed to the published version of the manuscript.

Funding: This research received no external funding.

Institutional Review Board Statement: The study was conducted according to the guidelines of the Declaration of Helsinki, reviewed and approved by the Medical Ethics Committee of 921 Hospital.

Informed Consent Statement: Informed consent was obtained from all subjects involved in the study. 
Data Availability Statement: The datasets presented in this article are not readily available because the datasets involve unfinished research projects. If necessary, requests to access the datasets should contact the corresponding author.

Acknowledgments: This research was supported by the Natural Science Foundation of China, Grant Number 62076248 and the Defense Industrial Technology Development Program, Grant Number JCKY2020550B003.

Conflicts of Interest: The authors have no potential conflict of interest to disclose.

\section{References}

1. Bastos, A.M.; Schoffelen, J.M. A tutorial review of functional connectivity analysis methods and their interpretational pitfalls. Front. Syst. Neurosci. 2016, 9, 175. [CrossRef]

2. Dailey, N.S.; Smith, R.; Vanuk, J.R.; Raikes, A.C.; Killgore, W.D. Resting-state functional connectivity as a biomarker of aggression in mild traumatic brain injury. Neuroreport 2018, 29, 1413-1417. [CrossRef]

3. Helfrich, R.F.; Knepper, H.; Nolte, G.; Sengelmann, M.; König, P.; Schneider, T.R.; Engel, A.K. Spectral fingerprints of large-scale cortical dynamics during ambiguous motion perception. Hum. Brain Mapp. 2016, 37, 4099-4111. [CrossRef] [PubMed]

4. Finn, E.S.; Shen, X.; Scheinost, D.; Rosenberg, M.D.; Huang, J.; Chun, M.M.; Papademetris, X.; Constable, R.T. Functional connectome fingerprinting: Identifying individuals using patterns of brain connectivity. Nat. Neurosci. 2015, 18, 1664-1671. [CrossRef]

5. Van, D.H.; Martijn, P.; Sporns, O. Network hubs in the human brain. Trends Cogn. Sci. 2013, 17, $683-696$.

6. Valizadeh, S.A.; Riener, R.; Elmer, S.; Jäncke, L. Decrypting the electrophysiological individuality of the human brain: Identification of individuals based on resting-state EEG activity. NeuroImage 2019, 197, 470-481. [CrossRef]

7. Bidgoly, A.J.; Bidgoly, H.J.; Arezoumand, Z. A survey on methods and challenges in EEG based authentication-ScienceDirect. Comput. Secur. 2020, 93, 101788. [CrossRef]

8. Shuang, L.; Bai, Y.; Jing, L.; Qi, H.; Ming, D. Individual Feature Extraction and Identification on EEG Signals in Relax and V-isual Evoked Tasks. Commun. Comput. Inf. Sci. 2014, 404, 305-318.

9. Höller, Y.; Uhl, A.; Bathke, A.; Thomschewski, A.; Butz, K.; Nardone, R.; Fell, J.; Trinka, E. Reliability of EEG measures of interaction: A paradigm shift is needed to fight the reproducibility crisis. Front. Hum. Neurosci. 2017, 11, 441. [CrossRef] [PubMed]

10. Maiorana, E.; La Rocca, D.; Campisi, P. On the Permanence of EEG Signals for Biometric Recognition. IEEE Trans. Inf. Forensics Secur. 2015, 11, 163-175. [CrossRef]

11. Chu, C.J.; Kramer, M.A.; Pathmanathan, J.; Bianchi, M.T.; Westover, M.B.; Wizon, L.; Cash, S.S. Emergence of Stable Functional Networks in Long-Term Human Electroencephalography. J. Neurosci. 2012, 32, 2703-2713. [CrossRef]

12. Fraschini, M.; Hillebrand, A.; Demuru, M.; Didaci, L.; Marcialis, G.L. An EEG-Based Biometric System Using Eigenvector Centrality in Resting State Brain Networks. IEEE Signal Process. Lett. 2014, 22, 666-670. [CrossRef]

13. Delorme, A.; Makeig, S. EEGLAB: An open source toolbox for analysis of single-trial EEG dynamics including independent component analysis. J. Neurosci. Methods 2004, 134, 9-21. [CrossRef] [PubMed]

14. Kayser, J.; Tenke, C.E. Principal components analysis of Laplacian waveforms as a generic method for identifying ERP generator patterns: I. Evaluation with auditory oddball tasks. Clin. Neurophysiol. 2006, 117, 369-380. [CrossRef] [PubMed]

15. Pascual-Marqui, R.D.; Michel, C.M.; Lehmann, D. Segmentation of brain electrical activity into microstates: Model estimation and validation. IEEE Trans. Biomed. Eng. 1995, 42, 658-665. [CrossRef]

16. Niso, G.; Bruña, R.; Pereda, E.; Gutiérrez, R.; Bajo, R.; Maestú, F.; Del-Pozo, F. HERMES: Towards an integrated toolbox to characterize functional and effective brain connectivity. Neuroinformatics 2013, 11, 405-434. [CrossRef]

17. He, B.; Astolfi, L.; Valdes-Sosa, P.A.; Marinazzo, D.; Palva, S.O.; Bénar, C.-G.; Michel, C.M.; Koenig, T. Electrophysiological Brain Connectivity: Theory and Implementation. IEEE Trans. Biomed. Eng. 2019, 66, 2115-2137. [CrossRef] [PubMed]

18. Escudero, J.; Hornero, R.; Abásolo, D. Interpretation of the auto-mutual information rate of decrease in the context of biomedical signal analysis. Application to electroencephalogram recordings. Physiol. Meas. 2009, 30, 187-199. [CrossRef]

19. Malmivuo, J.; Plonsey, R. Bioelectromagnetism. Med. Biol. Eng. Comput. 1996, 34, 9-12.

20. Syed, M.F.; Lindquist, M.A.; Pillai, J.J.; Agarwal, S.; Gujar, S.K.; Choe, A.S.; Caffo, B.; Sair, H.I. Dynamic functional connectivity states between the dorsal and ventral sensorimotor networks revealed by dynamic conditional correlation analysis of resting-state functional magnetic resonance imaging. Brain Connect. 2017, 7, 635-642. [CrossRef]

21. La Rocca, D.; Campisi, P.; Vegso, B.; Cserti, P.; Kozmann, G.; Babiloni, F.; Fallani, F.D.V. Human brain distinctiveness based on EEG spectral coherence connectivity. IEEE Trans. Biomed. Eng. 2014, 61, 2406-2412. [CrossRef] [PubMed]

22. Xia, M.; Wang, J.; He, Y. BrainNet Viewer: A network visualization tool for human brain connectomics. PLoS ONE 2013, 8, e68910. [CrossRef] [PubMed]

23. Maiorana, E.; Rocca, D.L.; Campisi, P. Eigenbrains and Eigentensorbrains: Parsimonious bases for EEG biometrics. Neurocomputing 2016, 171, 638-648. [CrossRef]

24. Kringelbach, M.L.; Deco, G. Brain States and Transitions: Insights from Computational Neuroscience. Cell Rep. 2020, $32,108128$. [CrossRef] 
25. Fries, P. A mechanism for cognitive dynamics: Neuronal communication through neuronal coherence. Trends Cogn. Sci. 2005, 9, 474-480. [CrossRef] [PubMed]

26. Rypma, B.; D'Esposito, M. The roles of prefrontal brain regions in components of working memory: Effects of memory load and individual differences. Proc. Natl. Acad. Sci. USA 1999, 96, 6558-6563. [CrossRef] [PubMed]

27. Cole, M.W.; Yarkoni, T.; Repovš, G.; Braver, T.S. Global connectivity of prefrontal cortex predicts cognitive control and intelligence. J. Neurosci. 2012, 32, 8988-8999. [CrossRef] [PubMed] 